\title{
Review of: "The monoamine stabilizer OSU6162 has anxiolytic-like properties and reduces voluntary alcohol intake in a genetic rat model of depression"
}

\author{
Andrea Godino \\ 1 Instituto Ferreyra
}

Potential competing interests: The author(s) declared that no potential competing interests exist.

Comments to the Authors

The present study focusses on the analysis of OSU6162 effects as pharmacological treatment of depression. Specifically, the authors give new information regarding its effect on anxiety responses since are missing data in the literature. This work was done carefully and described well, however, several concerns that the authors should address:

-In the novelty suppressed feeding test, the six hours of food restriction, were performed during light or dark cycle? The authors mentioned that all the test and behavioral measurement have been performed during dark cycle. I suggest clarified the food restriction period because the eating or hunger pattern of rat is very different depending the cycle of day.

-During the long period of the experiment there are some developmental changes that possibly also modified the OSU impact, the anxiety or ethanol intake. For example, the acceptance of a high concentration ethanol solution is different for adolescents and adults' rats and depend on previous experience. I recommend include some discussion of this aspect.

-The result of the study is very interesting and allow us to infer that another mechanism (possibly modulated by monoamines) is the neurobiological substrate and it is involved in these anxiolytic and alcohol consumption effects. Rationale: FSL rats have an anxious phenotype, therefore they drink more alcohol. Intermittent alcohol treatment modulates some neurobiological mechanism in these animals that reverses anxiety, they become more refractory to OSU treatment and behave as a control (FRL alcoholnaive, test 2). In contrast, FRL control animals do not respond to OSU when are naive. However, after intermittent treatment with alcohol, these rats behave like FSL (naive), being more anxious and being able to reverse this phenotype by OSU treatment (these effects did not reach to significant level in figure 4, and the figure 5 a show the same trend despite of the authors did not compare statistically the alcohol naive vs alcohol group??). Thus, the ethanol treatment increased the normal performance in altered rats (FSL) and also it altered normal or control rat. Considering also, that both strains of rat present the same alcohol intake pattern, in spite of water was also include during the test. OSU only has effects when the animals are more anxious. The authors include a discussion of this phenomenon; however, some others questions are released in this context: 
-The alcohol FSL group trend to decreased the anxiety after OSU but not reach significant levels (Fig 5a and c). Unfortunately, the authors could not use more animals for ethical reasons. What is the authors think about that, the doses of OSU have to be higher, the ontogenetical age of the rat or another explanation...

-The FRL rats were phenotypically altered (or they have an important tendency to that fig 4 and $5 a$ ) by the alcohol intake, this anxiety responses also return to baseline after OSU. Which neurobiological mechanisms postulate for this effect? They mentioned dopaminergic and other possibly systems. The discussion will be improved if they deep this aspect. It has been demonstrated that the acute and chronic alcohol exposure has an action on vasopressin and oxytocin hypothalamic neurons and its blood concentration. Also, an early experience with ethanol promotes an anxiety phenotype and later ethanol consumption. Both systems have been previously involved in the alcohol drinking and anxiety modulation. And oxytocinergic system also is implicated in the satiation process of feeding (Verbalis 1991, Bowen et al., 2011; Hammock, 2015; Cragg, Ji y Neugebauer, 2016; Godino et al 2015; Godino and Renard 2018; WilleBille et al., 2018). Regarding that, I would like to know if the author measured blood alcohol level previous NSF 2 in both trains of rats to evaluated a possible metabolic mechanism, or if they note body weight alteration after intermittent alcohol test between FSL and FRL rats. It has been demonstrated that also serotonergic system is able to modulate OT system (Jorgensen et al., 2003; Margatho et al., 2007).. I think that possibly monoamine stabilization by OSU reverted anxiety responses in both strains, being OSU a promising treatment for anxiety. 